\title{
Phase diagram of carbon nanotube ropes
}

\author{
J. González ${ }^{1}$ and J. V. Alvarez ${ }^{2}$ \\ ${ }^{1}$ Instituto de Estructura de la Materia, Consejo Superior de Investigaciones Científicas, Serrano 123, 28006 Madrid, Spain \\ ${ }^{2}$ Department of Physics, University of Michigan, Ann Arbor, Michigan 48109, USA
}

(Received 20 November 2003; published 13 July 2004)

\begin{abstract}
The zero-temperature phase diagram of carbon nanotube ropes is studied using a computational framework that incorporates the renormalization of intratube interactions and the effect of intertube Coulomb screening. This allows us to undertake a systematic analysis as a function of the number of metallic nanotubes in the rope and the effective strength of the phonon-mediated interaction. We find that there is in general a weak-coupling regime of the interactions, corresponding to Luttinger-liquid behavior in thin ropes and to superconducting behavior in sufficiently thick ropes. Furthermore, we show the existence of exotic phases in the strong-coupling regime, characterized by the appearance of charge instabilities that depend on the helicity and the doping level of the nanotubes in the rope. Our approach allows for the simultaneous analysis of the scaling of the Cooperpair tunneling amplitude between metallic nanotubes, making it possible to discern the crossover from purely one-dimensional physics to the setting of three-dimensional Cooper-pair coherence. We provide then good estimates of the superconducting transition temperature and discuss the connection of our results with recent experiments.
\end{abstract}

DOI: 10.1103/PhysRevB.70.045410

PACS number(s): 71.20.Tx, 71.10.Pm, 74.50. $+\mathrm{r}$

\section{INTRODUCTION}

Carbon nanotubes offer a great potential for technological applications. The fact that they can show metallic or semiconducting behavior depending on the helicity of the tubule ${ }^{1}$ makes them very suitable for the construction of devices in molecular electronics. Carbon nanotubes offer also an ideal ground to study the effects of electronic correlations, ${ }^{2-5}$ which are enhanced in systems with reduced dimensionality. Given that the conduction takes place in a one-dimensional (1D) structure, it has been proposed that the nanotubes should be ideal systems for the observation of the so-called Luttinger-liquid behavior. For contacts with low transparency, genuine signatures of Luttinger-liquid behavior have been observed in the power-law dependence of the conductance around room temperature. ${ }^{6,7}$ These experiments seem therefore to probe a regime in which the repulsive Coulomb interaction turns out to be dominant in the nanotubes.

There have been also experiments revealing the existence of superconducting (SC) correlations in the nanotubes. ${ }^{89}$ The observations reported in Ref. 8 were made in nanotube ropes suspended between SC electrodes, the most remarkable signal being the appearance of supercurrents for temperatures below the critical value of the contacts. What has been measured in that experiment is the proximity effect, by which Cooper pairs are formed in the nanotubes near the SC contacts. A consistent explanation of the behavior of the supercurrents measured experimentally has been proposed in Ref. 10 , stressing the interpretation of the supercurrents as an effect of 1D transport along the carbon nanotubes.

Moreover, SC transitions have been observed in ropes suspended between metallic, nonsuperconducting electrodes. ${ }^{11}$ In certain samples, a drop of several orders of magnitude in the resistance has been measured, showing the onset of a SC transition in the system with a finite number of channels. The measurements reported in Refs. 11 and 12 point at the existence of a SC phase intrinsic to the carbon nanotubes. More recently, strong SC correlations have been also reported in individual nanotubes of very short radius, inserted in a zeolite matrix. ${ }^{13}$ In that kind of experiment, the effects studied have 1D character, but clear superconductivity features have been obtained from the tendency to expel the magnetic flux and the divergent behavior of the conductance.

The observation of SC correlations implies the existence of an attractive component of the interaction in the carbon nanotubes. The analyses of the electron correlations in the tubules have shown that it is not plausible the opening of an attractive channel arising from the purely repulsive Coulomb interaction, at least without going down to extremely low energies. ${ }^{3,4}$ Then, it is most likely that the attractive interaction comes from the coupling to the elastic modes of the nanotube lattice. ${ }^{10}$ How the large Coulomb repulsion present in the nanotubes may be overcome by the attraction due to the phonon exchange remains the question. By means of a simple estimate, it can be seen that the strength of the latter is much smaller than the nominal strength of the Coulomb interaction, for nanotubes with the typical radii found in the ropes. It has been shown, however, that the electrostatic coupling between a large number of metallic nanotubes leads to a substantial reduction of the repulsive interaction within each nanotube. ${ }^{14,15}$ The origin of this effect is similar to that of screening in a three-dimensional (3D) conductor. In a rope, however, the single-electron tunneling amplitude between neighboring metallic nanotubes is highly suppressed in general, due to the misalignment of the respective carbon lattices. ${ }^{16,17}$ The absence of a significant intertube electron hopping is what keeps the Coulomb interaction long ranged in the nanotubes, despite the large reduction of its strength in the thick ropes.

A microscopic model has been already proposed to account for the observation of superconductivity intrinsic to the ropes of nanotubes. ${ }^{14}$ It has been argued that, as long as the Cooper pairs are formed at zero total momentum, they do not 
find the obstacle that single electrons have to tunnel between neighboring nanotubes due to the misalignment of the lattices. The existence of a SC transition is possible in the ropes as the coherence in the transverse directions is established through the tunneling of Cooper pairs between the nanotubes. ${ }^{14}$ In this approach, the balance between the Coulomb repulsion and the effective attraction coming from phonon exchange has been studied in forward-scattering channels, where the electrostatic coupling between the metallic nanotubes plays the most significant role.

In this paper we refine substantially the theoretical construction proposed to explain the superconductivity of the nanotube ropes. This is accomplished by incorporating the backscattering and Umklapp processes that arise from phonon-mediated interactions. ${ }^{15}$ These provide in general larger contributions than those from the Coulomb interaction to that kind of processes. For this reason, there is first the question of reexamining at which scale the interactions in the backscattering and Umklapp channels are able to destabilize the Luttinger-liquid picture of the carbon nanotubes, and the kind of phases that may appear under those perturbations.

We undertake a most accurate description of the nanotube ropes by implementing a computational framework that deals altogether with the electrostatic coupling between the metallic nanotubes and the renormalization of the intratube interactions, which are enhanced at low energies. ${ }^{15}$ This makes it possible to discern between the electronic properties of the ropes at weak coupling and at strong coupling, as the instabilities of the electron system are different in each case. At weak coupling, we will see that the picture already elaborated using bosonization methods is in essence correct, accounting for a regime of Luttinger-liquid behavior in thin ropes and for a SC phase in sufficiently thick ropes. Furthermore, we will find new exotic phases at strong coupling, characterized by the appearance of charge instabilities that depend on the helicity and the doping level of the nanotubes in the rope.

We will rely on the analysis of the scaling of the different interactions at low energies in our aim of getting insight into the electronic instabilities of the nanotube ropes. This method has the advantage of allowing the simultaneous analysis of the scaling of the Cooper pair tunneling amplitude between metallic nanotubes. This is one of the key ingredients of our computational procedure, since it discriminates the regimes where a 3D phase-coherent state may be formed through the rope. Our results will make it possible to establish a fair comparison with the transition temperatures measured experimentally and to locate the position of the corresponding samples in our phase diagram.

\section{PHONON-MEDIATED INTERACTIONS IN CARBON NANOTUBES}

We consider first the effective electron-electron interactions that are generated by the exchange of phonons between electronic currents. As we are going to see, this demands a careful analysis, since the repulsive or attractive character of the interaction in each particular channel depends on the geometry of the nanotube. In general, the exchange of phonons gives rise to a retarded interaction, which can be represented by the effective potential obtained after integrating out the phonons in the many-body theory. These are bound to propagate then between electron-phonon vertices, which have couplings $g\left(k, k^{\prime}\right)$ depending on the momenta of the incoming and outgoing electrons. The effective potential bears this dependence on the momenta as well as on the frequency $\omega$, turning out to be

$$
V(\omega)=-g\left(k, k^{\prime}\right) g\left(q, q^{\prime}\right) \frac{\omega_{k-k^{\prime}}}{-\omega^{2}+\omega_{k-k^{\prime}}^{2}},
$$

where $\omega_{k-k^{\prime}}$ stands for the energy of the exchanged phonon with momentum $k-k^{\prime}=q^{\prime}-q \cdot{ }^{18}$

It is seen from Eq. (1) that the retarded interaction usually becomes attractive at frequencies below the characteristic phonon energy. In this respect, the acoustic phonons with dispersion depending linearly on momentum do not lead to a significant energy range of attraction. This comes from the fact that the sound velocity $v_{s}$ in the nanotubes is more than 40 times smaller than the Fermi velocity $v_{F}$. The exchange of acoustic phonons at low momentum transfer produces only a very weak enhancement of the electron correlations, in the form of contributions to the critical exponents that deviate from the noninteracting behavior by corrections of the order of $\left(v_{s} / v_{F}\right)^{2}$, as shown in Ref. 19. The influence of the acoustic phonons is therefore negligible at low momentum transfer regarding the $\mathrm{SC}$ effects in carbon nanotubes. It can be easily seen that a straightforward extension of the argument leads to the same conclusion for other low-energy modes at the bottom of the phonon spectrum.

The most significant role in the development of the SC correlations has to be played by high-energy phonons and, in particular, by the modes of the optical branches of the spectrum. These are found up to energies of the order of $0.2 \mathrm{eV},{ }^{20-22}$ which are comparable to those of the low-energy electron modes relevant for the 1D transport in the carbon nanotubes. The optical phonon modes correspond to localized displacements of the nanotube lattice. This makes appropriate the use of a tight-binding approximation for the calculation of the electron-phonon couplings in Eq. (1). In the carbon nanotubes, the couplings also bear an explicit dependence on the subbands $p$ and $p^{\prime}$ (to be described later) to which the incoming and outgoing electron modes belong, and they can be represented by a sum over nearest neighbors of the atoms in the unit cell of the nanotube ${ }^{23}$

$$
\begin{aligned}
g_{p, p^{\prime}}\left(k, k^{\prime}\right)= & \frac{1}{\left(\mu \omega_{k-k^{\prime}}\right)^{1 / 2}} \sum_{\left\langle s, s^{\prime}\right\rangle} u_{s}^{(p)^{*}}(k) u_{s^{\prime}}^{\left(p^{\prime}\right)}\left(k^{\prime}\right)\left[\epsilon_{s}\left(k-k^{\prime}\right)\right. \\
& \left.-\epsilon_{s^{\prime}}\left(k-k^{\prime}\right)\right] \cdot \nabla J\left(s, s^{\prime}\right),
\end{aligned}
$$

where $\mu$ is the mass per unit length, $\epsilon_{s}\left(k-k^{\prime}\right)$ is the phonon polarization vector at site $s$, and $u_{s^{\prime}}^{\left(p^{\prime}\right)}\left(k^{\prime}\right), u_{s}^{(p)}(k)$ are the respective amplitudes of the incoming and outgoing electrons. $J\left(s, s^{\prime}\right)$ is the matrix element of the atomic potential between nearest-neighbor orbitals at sites $s$ and $s^{\prime}$, so that the gradient in Eq. (2) accounts for the variation of the matrix element 


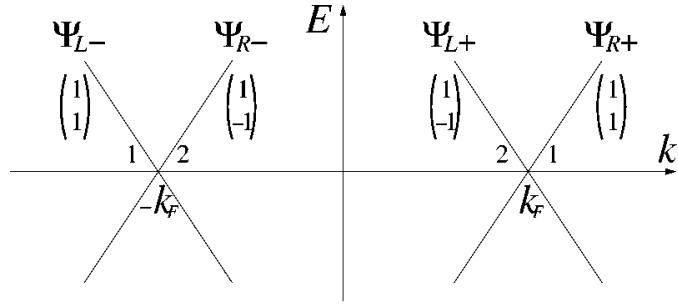

(a)
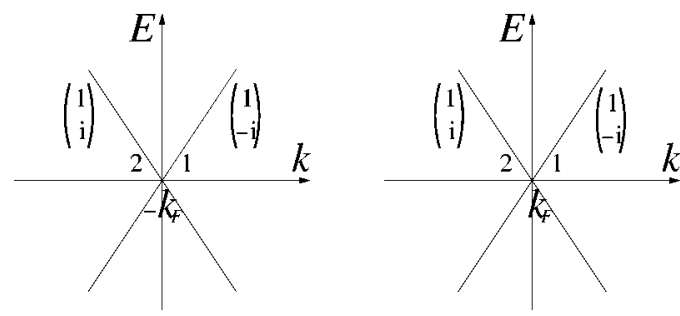

(b)

FIG. 1. Scheme of the low-energy linear branches of armchair nanotubes (upper figure) and metallic zigzag nanotubes (lower figure) crossing at opposite momenta $k_{F}$ and $-k_{F}$. The spinors correspond to the relative electron amplitudes in the two sublattices of graphene rolled up to form the nanotube. The labels 1 and 2 at each linear branch are used to identify the corresponding subband according to the symmetry of the spinors.

under small relative displacements of the orbitals with respect to the equilibrium position. ${ }^{24}$

The influence of the nanotube geometry enters through the specific symmetry of the electron modes $u_{s}^{(p)}(k)$ in the gapless subbands, which depends on the helicity of the tubule. We are going to deal in particular with the cases of armchair and zigzag nanotubes, since any other geometry for a chiral nanotube can be thought as interpolating between those two ideal instances. We recall that the low-energy spectrum of any metallic nanotube is given by two pairs of linear branches crossing at opposite points in momentum space, as shown in Fig. 1. The linear branches correspond actually to the different eigenvalues of a one-particle Hamiltonian depending on the longitudinal component $k$ of the momentum and operating in the two-atom basis of the nanotube lattice 25

$$
\mathcal{H}=v_{F}\left(\begin{array}{cc}
0 & \alpha k \\
\alpha^{*} k & 0
\end{array}\right),
$$

where $v_{F}$ is the Fermi velocity and $\alpha$ is a complex parameter interpolating between 1 and $i$ for armchair and zigzag nanotubes, respectively.

Each linear branch in a metallic nanotube has therefore its corresponding eigenvector that binds the relative electron amplitudes in the atoms of the lattice basis. The expressions for the two linear branches at each Fermi point take in general the form

$$
\left(\begin{array}{c}
1 \\
e^{i \phi}
\end{array}\right),\left(\begin{array}{c}
1 \\
-e^{i \phi}
\end{array}\right),
$$

where $\phi$ is an angle that vanishes for armchair nanotubes and equals $\pi / 2$ for zigzag nanotubes. The different symmetry of the eigenvectors depending on the particular linear branch is represented in Fig. 1 for those two particular geometries. The linear branches can be ascribed in general to any of two different subbands $p=1,2$, which correspond to the different form of the eigenvectors in Eq. (4).

The form of the relative electron amplitudes in the two atoms of the lattice basis affects directly the evaluation of the electron-phonon couplings, since these are given by a sum over nearest-neighbor sites of the carbon lattice. The fact that the only difference between the two eigenvectors shown in Eq. (4) is a relative - sign translates, for instance, into a precise relation between the electron-phonon couplings involving the linear branches with different symmetry. Recalling our notation for the subband label $p=1,2$, we have

$$
\begin{aligned}
& g_{1,1}\left(k, k^{\prime}\right)=-g_{2,2}\left(k, k^{\prime}\right), \\
& g_{1,2}\left(k, k^{\prime}\right)=-g_{2,1}\left(k, k^{\prime}\right) .
\end{aligned}
$$

These relations just follow from the properties of the sum in Eq. (2) under the exchange of modes with different symmetry, in such a way that they hold irrespective of the particular form of the phonons involved.

There is moreover an important set of selection rules that can be derived from the expression (2), and which depend on the particular kinematics of the scattered electron modes and on the geometry of the nanotube. ${ }^{26}$ In the case of armchair nanotubes, for instance, we see from Eq. (4) that the modes in the bonding subband have the same amplitude in the two atoms of the lattice basis, while those in the antibonding subband have opposite amplitudes. Then, if the incoming and outgoing electrons have opposite momenta, it follows from expression (2) that the terms in the sum related by the exchange of the sites $s$ and $s^{\prime}$ just differ by a - sign, when the in and out electron modes belong to different subbands. We conclude that

$$
g_{1,2}(k,-k)=0
$$

in the case of the armchair nanotubes. ${ }^{26}$ An explicit realization of this property can be seen in the expression of the electron-phonon coupling for transverse acoustic phonons obtained in Ref. 23.

In the case of the zigzag nanotubes, the two eigenvectors in Eq. (4) are mutually complex conjugate. Then, under the exchange of the sites $s$ and $s^{\prime}$ in the expression (2), the different terms in the sum remain unaltered, when the in and out electron modes belong to different subbands. This implies that the rule (7) is not at work in that case. Instead, it can be easily shown that the terms in the sum change sign under the exchange of the nearest-neighbor sites when the electron modes belong to the same subband, i.e., to linear branches with the same eigenvector symmetry. Thus we have

$$
g_{1,1}(k,-k)=g_{2,2}(k,-k)=0
$$

in the case of the zigzag nanotubes. ${ }^{26}$

The vanishing of the electron-phonon couplings in Eqs. (7) and (8) reflects actually their odd behavior as a function of the sum of the momenta for the incoming and outgoing electrons. This property can be derived from the representation (2), and it is also apparent in the particular expression of 
the coupling for acoustic phonons in the armchair nanotubes derived in Ref. 23. We may conclude that the electronphonon scattering amplitudes involving that kind of couplings must average to zero when the in and out electron modes are about opposite points in momentum space. This is an usual situation when the nanotubes are at half filling, since then the Fermi level is right at the crossing points of the linear branches.

The constraints given by Eqs. (5)-(8) have important consequences when they are translated into the effective electron-electron interactions built from Eq. (1). We start this analysis by classifying these into different channels with respective coupling constants $g_{i}^{(j)} .{ }^{27}$ The lower index discerns whether the interacting particles shift from one Fermi point to the other $(i=1)$, remain at different Fermi points $(i=2)$, or they interact near the same Fermi point $(i=4)$. The upper label follows the same rule to classify the different combinations of left movers and right movers, including the possibility of having Umklapp processes $(j=3)$.

Using the general property

$$
g_{p, p^{\prime}}^{*}\left(k, k^{\prime}\right)=g_{p^{\prime}, p}\left(k^{\prime}, k\right),
$$

it can be shown that all the interactions of backscattering type mediated by phonons have attractive character. This comes from the fact that the product of the two electronphonon couplings in Eq. (1) is then a positive quantity, so that the potential gets negative values for frequencies below the characteristic phonon energy. Therefore, the contributions from phonon exchange processes (that we will denote with the label ex) are negative in the case of the couplings $g_{i}^{(1)}$, that stand for interactions in which the incoming modes exchange their left- and right-moving character

$$
g_{1, \mathrm{ex}}^{(1)}<0, g_{2, \mathrm{ex}}^{(1)}<0, g_{4, \mathrm{ex}}^{(1)}<0 .
$$

In the processes contributing to $g_{1}^{(2)}$, the interacting electrons retain their left- and right-moving character while, in those contributing to $g_{1}^{(4)}$, the electrons remain both left moving or right moving. The phonon-exchange processes average to zero for both couplings in the case of undoped nanotubes, since they involve electron-phonon couplings with the kinematics implying Eqs. (7) and (8). The interaction is attractive in those channels away from half filling, although the respective couplings turn out to be proportional to the square of the doping rate. ${ }^{28}$

On the other hand, the Umklapp processes contributing to the couplings $g_{i}^{(3)}$ are characterized by the fact that the interacting electrons shift from left to right moving, or vice versa. When mediated by phonon exchange, these processes are related to respective backscattering processes through the exchange of the subbands 1 and 2 in one of the electronphonon couplings. This operation introduces a relative minus sign between the respective scattering amplitudes, according to the relations (5) and (6). Therefore, the effective interaction becomes repulsive in the channels corresponding to the couplings $g_{1}^{(3)}, g_{2}^{(3)}$, and $g_{4}^{(3)}$, which get contributions from phonon exchange

$$
g_{1, \mathrm{ex}}^{(3)}>0, g_{2, \mathrm{ex}}^{(3)}>0, g_{4, \mathrm{ex}}^{(3)}>0 .
$$

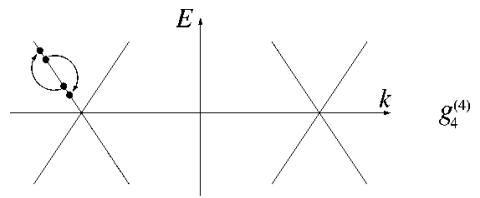

(a)

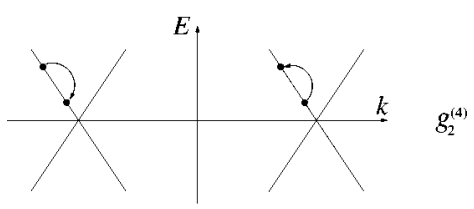

(b)

FIG. 2. Small momentum-transfer processes corresponding to the couplings $g_{4}^{(4)}$ and $g_{2}^{(4)}$.

Regarding the interactions mediated by phonons in the forward-scattering channels $g_{4}^{(4)}, g_{4}^{(2)}, g_{2}^{(2)}$, and $g_{2}^{(4)}$, they may be attractive or repulsive, depending on the geometry of the nanotube and on whether it is doped or not. In the case of the undoped zigzag nanotubes, the electron-phonon amplitudes involved are odd functions of the sum of the longitudinal momenta for the incoming and the outgoing electrons, according to our discussion below Eq. (8). Since each interacting electron remains around a given Fermi point with longitudinal momentum $k=0$ at half filling, this means that the contributions by phonon exchange to the forward-scattering couplings $g_{4}^{(4)}, g_{4}^{(2)}, g_{2}^{(2)}$, and $g_{2}^{(4)}$ must average to zero in the undoped zigzag nanotubes. ${ }^{26}$

Away from half filling, the kinematical constraints that lead to the selection rules (8) are not at work in the zigzag nanotubes. The forward-scattering couplings get nonvanishing contributions from phonon exchange, although they are reduced by a factor proportional to the square of the doping rate. The processes that take place within the same linear branch give rise to effective attraction in the channels corresponding to $g_{4}^{(4)}$ and $g_{2}^{(4)}$, shown in Fig. 2. By using the complex conjugate relation between modes in opposite linear branches of a zigzag nanotube, it is easy to show that the product of electron-phonon couplings in Eq. (1) is always positive for contributions to the $g_{4}^{(2)}$ and $g_{2}^{(2)}$ couplings represented in Fig. 3, so that the effective interaction also becomes attractive in those channels.

In the case of the undoped armchair nanotubes, there are no selection rules that may constrain the forward-scattering processes. The interactions mediated by phonons in the channels corresponding to the $g_{4}^{(4)}$ and $g_{2}^{(2)}$ couplings are always attractive, since they involve the scattering between electrons in the same subband of the armchair nanotube. This is the other way around for the processes contributing to the $g_{4}^{(2)}$ and $g_{2}^{(4)}$ couplings, since the scattering is then between an electron in the bonding subband and another in the antibonding subband. Consequently, the two electron-phonon couplings that appear in Eq. (1) have to differ by a relative minus sign. Thus, the contributions by phonon exchange to the $g_{4}^{(2)}$ and $g_{2}^{(4)}$ couplings are positive for frequencies below the characteristic phonon energy, so that the effective interaction becomes repulsive in the corresponding channels. 


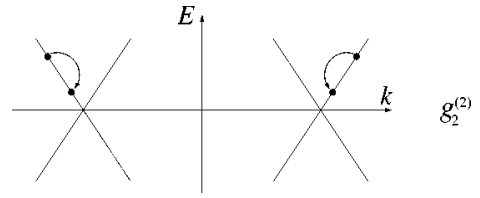

(a)

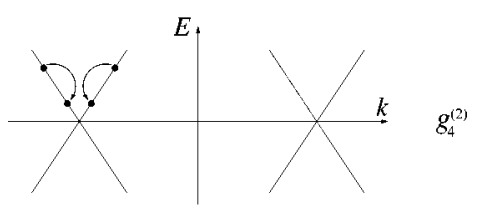

(b)

FIG. 3. Small momentum-transfer processes corresponding to the couplings $g_{2}^{(2)}$ and $g_{4}^{(2)}$.

These properties hold for the armchair nanotubes, irrespective of whether the electron system is doped or not.

In order to compare later with the repulsive Coulomb interaction, we can make an estimate of the strength of the effective interaction by phonon exchange. We will take henceforth the limit in which the interaction mediated by Eq. (1) becomes instantaneous. The order of magnitude of the potential is then given by the variation of the matrix element $J$ with the C-C distance $a, \partial J / \partial a \approx 4.5 \mathrm{eV} \AA^{-1}$, and the characteristic energy scale at the top of the phonon spectrum, $\omega_{k} \approx 0.2 \mathrm{eV}$. In this respect, the energy of phonons in processes with momentum transfer $2 k_{F}$ becomes comparable to that of the optical phonons at low momentum transfer, so that we can take a common strength $g$ with

$$
|g| \sim(\partial J / \partial a)^{2} / \mu \omega_{k}^{2}
$$

in all the channels of the effective interaction. Taking the linear mass density $\mu$ appropriate for typical nanotubes with diameter around $1.4 \mathrm{~nm}$, we find that $|g|$ turns out to be of the order of $0.1 v_{F}$, in terms of the Fermi velocity $v_{F} \approx 8$ $\times 10^{5} \mathrm{~ms}^{-1}$.

\section{COULOMB INTERACTIONS AND LUTTINGER LIQUID REGIME}

Now we turn our attention to the Coulomb interaction and the confrontation with the effective interaction mediated by phonon exchange. In one spatial dimension there is no conventional plasmon screening of the Coulomb interaction, that therefore remains long ranged. ${ }^{29,30}$ The Coulomb potential $V_{C}(k)$ can be parametrized with two system-dependent parameters $^{31}$

$$
V_{C}(k)=V_{0} \ln \left(\frac{k_{c}+k}{k}\right),
$$

where $V_{0}$ encodes the intensity of the interaction and $k_{c}$ is a momentum cutoff determined by the system geometry. For the carbon nanotubes, $k_{c}$ is of the order of the inverse of the nanotube radius $R$, as it is the memory that the electron system keeps of the finite transversal size, after projection of the $3 \mathrm{D}$ potential onto the longitudinal direction of the tubule.
Expression (13) interpolates between the two well-known limits of the 1D Coulomb potential $V(k) \rightarrow \ln (1 / k)$ as $k \rightarrow 0$ and $V(k) \rightarrow 1 / k$ if $k \gg k_{c}$.

The role of the Coulomb interaction in an isolated metallic nanotube has been the subject of intense study, specially in relation to the experimental observation of Luttingerliquid phenomenology. Actually, it has been shown ${ }^{2-4}$ that the strength of the backscattering and Umklapp processes mediated by the Coulomb interaction is reduced by a relative factor $\sim 0.1 a / R$, in terms of the ratio of the C-C distance $a$ to the nanotube radius $R$, with respect to the nominal strength of the potential in Eq. (13). Therefore, for typical nanotubes with a diameter about $1.4 \mathrm{~nm}$, the Coulomb contribution to backscattering and Umklapp scattering can be safely neglected in favor of the contributions from phonon exchange, whenever the strength $|g|$ of the latter is above $\sim 0.05 v_{F}$. We have explicitly checked this point by studying the sensitivity of our results to small changes in these couplings.

The competition between the long-range Coulomb interaction and the effective interaction from phonon exchange takes place in the forward-scattering channels. These correspond to the couplings $g_{2}^{(2)}, g_{2}^{(4)}, g_{4}^{(2)}$, and $g_{4}^{(4)}$. The coefficient in front of the potential (13) turns out to be of order 1 times the Fermi velocity $v_{F}$, after introducing a value of the dielectric constant appropriate for typical experimental conditions. ${ }^{3}$ Moreover, the Coulomb interaction is further enhanced at low momentum transfer due to the logarithmic dependence of the potential. Taking into account the above estimate for the strength of the phonon-exchange interaction in nanotubes of typical thickness, it becomes clear that the Coulomb repulsion overcomes by far that source of attraction in the forward-scattering channels, in the case of isolated singlewalled nanotubes.

An isolated metallic nanotube can be described then by a $1 \mathrm{D}$ model of interacting electrons in which the $g_{2}^{(2)}, g_{2}^{(4)}, g_{4}^{(2)}$, and $g_{4}^{(4)}$ couplings are much larger in absolute value than those for backscattering and Umklapp interactions. As a first approximation, we can focus on the model with just the four forward-scattering couplings, which has the advantage of leading to a completely integrable system. At this stage, switching on the interaction produces a profound reorganization of the single-particle spectrum. The free-electron description is unstable against electron-electron interactions. $^{32,33}$ To remain metallic, the $1 \mathrm{D}$ system has to suppress spectral weight at the Fermi level forming a liquid with a power-law-like $\omega^{\alpha}$ spectral density. This characterizes the Luttinger-liquid regime of the carbon nanotubes, in which the correlation functions and different observables have power-law behavior governed by suitable critical exponents. ${ }^{3,4}$

The forward-scattering interactions can be written in terms of the electron density operators

$$
\rho_{r i \sigma}(x)=\Psi_{r i \sigma}^{\dagger}(x) \Psi_{r i \sigma}(x)
$$

which correspond to the different electron fields $\Psi_{\text {rio }}$ for the linear branches shown in Fig. 1. We adopt a notation in which the index $r=L, R$ is used to label the left- or rightmoving character of the linear branch, and the index $i= \pm$ to 
label the Fermi point. The index $\sigma$ stands for the two different spin projections. As the interaction by phonon exchange and the Coulomb interaction do not depend on the spin of the interacting electrons, we may carry out the discussion in terms of the charge density operators

$$
\rho_{\text {ric }}(k)=\frac{1}{\sqrt{2}}\left[\rho_{r i \uparrow}(k)+\rho_{r i \downarrow}(k)\right] .
$$

Moreover, it is convenient to define the symmetric and antisymmetric combinations of the corresponding density operators in the two Fermi points

$$
\begin{aligned}
& \tilde{\rho}_{R S c}(k)=\frac{1}{\sqrt{2}}\left[\rho_{R+c}(k)+\rho_{R-c}(k)\right], \\
& \tilde{\rho}_{R A c}(k)=\frac{1}{\sqrt{2}}\left[\rho_{R+c}(k)-\rho_{R-c}(k)\right], \\
& \tilde{\rho}_{L S c}(k)=\frac{1}{\sqrt{2}}\left[\rho_{L-c}(k)+\rho_{L+c}(k)\right], \\
& \tilde{\rho}_{L A c}(k)=\frac{1}{\sqrt{2}}\left[\rho_{L-c}(k)-\rho_{L+c}(k)\right] .
\end{aligned}
$$

With this change of variables, the Hamiltonian for the forward-scattering interactions can be written in the form

$$
\begin{aligned}
H_{\mathrm{FS}}= & \frac{1}{2} v_{F} \int_{-k_{c}}^{k_{c}} d k \sum_{r i \sigma}: \rho_{r i \sigma}(k) \rho_{r i \sigma}(-k):+\frac{1}{2} \int_{-k_{c}}^{k_{c}} \frac{d k}{2 \pi} 2\left[\tilde{\rho}_{R S c}(k)\right. \\
& \times\left(g_{4}^{(4)}+g_{2}^{(4)}\right) \widetilde{\rho}_{R S c}(-k)+\widetilde{\rho}_{L S c}(k)\left(g_{4}^{(4)}+g_{2}^{(4)}\right) \widetilde{\rho}_{L S c}(-k) \\
& +\widetilde{\rho}_{R A c}(k)\left(g_{4}^{(4)}-g_{2}^{(4)}\right) \widetilde{\rho}_{R A c}(-k)+\widetilde{\rho}_{L A c}(k)\left(g_{4}^{(4)}-g_{2}^{(4)}\right) \\
& \times \widetilde{\rho}_{L A c}(-k)+2 \widetilde{\rho}_{R S c}(k)\left(g_{2}^{(2)}+g_{4}^{(2)}\right) \tilde{\rho}_{L S c}(-k) \\
& \left.+2 \widetilde{\rho}_{R A c}(k)\left(g_{2}^{(2)}-g_{4}^{(2)}\right) \widetilde{\rho}_{L A c}(-k)\right],
\end{aligned}
$$

where $k_{c}$ stands again for the momentum cutoff dictated by the transverse size of the system.

We see that the symmetric and the antisymmetric combination of the charge density near the two Fermi points are completely decoupled in the Hamiltonian (20). The quadratic expression in the density operators can be diagonalized separately in the two sectors. Thus, an individual metallic nanotube can be characterized at this stage by four independent velocities featuring the Luttinger-liquid behavior ${ }^{32,33}$

$$
\begin{aligned}
& v_{N \pm}=v_{F}+\frac{g_{4}^{(4)} \pm g_{2}^{(4)}}{\pi}+\frac{g_{2}^{(2)} \pm g_{4}^{(2)}}{\pi}, \\
& v_{J \pm}=v_{F}+\frac{g_{4}^{(4)} \pm g_{2}^{(4)}}{\pi}-\frac{g_{2}^{(2)} \pm g_{4}^{(2)}}{\pi} .
\end{aligned}
$$

In terms of these fundamental parameters, we can express several physical magnitudes of interest such as the renormalized velocities $u_{ \pm}$and the stiffnesses $K_{ \pm}$:

$$
K_{ \pm}=\sqrt{\frac{v_{J \pm}}{v_{N \pm}}},
$$

$$
u_{ \pm}=\sqrt{v_{N \pm} v_{J \pm}} \cdot
$$

These parameters govern in turn the thermodynamic and transport properties, encoded into the compressibilities $\kappa_{ \pm}$, the Drude weights $D_{ \pm}$and the dependence of the specific heat $C_{v}$ on the temperature $T$ :

$$
\begin{gathered}
\kappa_{ \pm}=\frac{2}{\pi v_{N \pm}}, \\
D_{ \pm}=2 v_{J \pm}, \\
\frac{C_{v}}{T}=\frac{\pi}{3}\left(\frac{1}{u_{+}}+\frac{1}{u_{-}}\right) .
\end{gathered}
$$

As already remarked, the description in terms of the forward-scattering interactions provides a good approximation to the behavior of isolated nanotubes, for which the Coulomb interaction is not significantly screened. ${ }^{3,4}$ The Coulomb repulsion gives then the dominant contribution to the couplings $g_{4}^{(4)}, g_{2}^{(2)}, g_{2}^{(4)}$, and $g_{4}^{(2)}$. This is consistent with the experimental observations of Luttinger-liquid behavior in the carbon nanotubes. ${ }^{6,7}$ However, one has to bear in mind that the liquid with just forward-scattering interactions is not stable under the perturbations introduced by the backscattering and the Umklapp scattering. Thus, although the strength of the latter may be nominally small compared to the Coulomb repulsion, their effects are enhanced as the system is probed at progressively low energies. The 1D electron system cannot support the transition to a phase with long-range order, but there is a tendency of the Luttinger liquid to break down, which can be materialized when any of the parameters (21) or (22) vanishes.

It is then important to have an estimate of the energy scale at which an individual nanotube may enter a new phase, either by the vanishing

$$
K_{+}=0 \text { or } K_{-}=0
$$

or the divergence

$$
1 / K_{+}=0 \text { or } 1 / K_{-}=0
$$

of any of the charge stiffnesses. A powerful tool to keep track of such dramatic changes is the renormalization group. ${ }^{32,33}$ From the technical point of view, the instabilities of the electron system manifest in the logarithmic dependence on energy of certain diagrams, that originates scaling relations between the couplings. These equations for individual nanotubes were derived to the one-loop order in Ref. 27. Our aim is to use them now to find the instabilities triggered by the effective backscattering and Umklapp interactions arising from phonon exchange.

The growth of the effective interactions at low energies can be understood from the fact that their couplings get anomalous dimensions, some of them depending on the forward-scattering couplings. We have, for instance, the scaling equations (up to terms quadratic in the backscattering and Umklapp couplings)

$$
\partial g_{1}^{(2)} / \partial l=\left(1-\frac{1}{K_{-}}\right) g_{1}^{(2)}+\frac{1}{\pi v_{F}}\left(g_{4}^{(3)} g_{1}^{(3)}-g_{2}^{(1)} g_{1}^{(1)}\right),
$$


TABLE I. Initial values of the couplings, as deduced in the text, for the scaling equations corresponding to an individual metallic nanotube. The quantity $v=\left(e^{2} / 2 \pi \kappa\right) \ln \left|k_{c} / k_{0}\right|$ is the average strength of the Coulomb potential over long distances, with $k_{0} \sim 10^{-3} k_{c}$ and $\kappa$ being a suitable dielectric constant. The quantity $g<0$ represents the contribution from phonon-exchange processes. The factor $\delta$ stands for the reduction proportional to the square of the doping rate in doped nanotubes.

\begin{tabular}{lcccc}
\hline \hline & \multicolumn{2}{c}{ armchair } & \multicolumn{2}{c}{ zigzag } \\
parameter & undoped & doped & undoped & doped \\
\hline$g_{1}^{1}$ & $g$ & $g$ & $g$ & $g$ \\
$g_{1}^{2}$ & 0 & $\delta g$ & 0 & $\delta g$ \\
$g_{1}^{3}$ & $-g$ & 0 & $-g$ & 0 \\
$g_{1}^{4}$ & 0 & $\delta g$ & 0 & $\delta g$ \\
$g_{2}^{1}$ & $g$ & $g$ & $g$ & $g$ \\
$g_{2}^{3}$ & $-g$ & 0 & $-g$ & 0 \\
$g_{4}^{1}$ & $g$ & $g$ & $g$ & $g$ \\
$g_{4}^{3}$ & $-g$ & 0 & $-g$ & 0 \\
$g_{2}^{2}$ & $v+g$ & $v+g$ & $v$ & $v+\delta g$ \\
$g_{2}^{4}$ & $v-g$ & $v-g$ & $v$ & $v+\delta g$ \\
$g_{4}^{2}$ & $v-g$ & $v-g$ & $v$ & $v+\delta g$ \\
$g_{4}^{4}$ & $v+g$ & $v+g$ & $v$ & $v+\delta g$ \\
\hline \hline
\end{tabular}

$\partial g_{1}^{(3)} / \partial l=\left(1-K_{+}\right) g_{1}^{(3)}+\frac{1}{\pi v_{F}}\left(-2 g_{1}^{(3)} g_{1}^{(1)}+g_{2}^{(3)} g_{1}^{(1)}+g_{4}^{(3)} g_{1}^{(2)}\right)$,

$$
\begin{aligned}
\partial g_{2}^{(1)} / \partial l= & \left(1-\frac{1}{K_{-}}\right) g_{2}^{(1)}+\frac{1}{\pi v_{F}}\left(g_{4}^{(1)} g_{1}^{(2)}-2 g_{4}^{(1)} g_{2}^{(1)}+g_{4}^{(3)} g_{1}^{(3)}\right. \\
& \left.-g_{4}^{(3)} g_{2}^{(3)}-g_{1}^{(2)} g_{1}^{(1)}\right) \\
\partial g_{2}^{(3)} / \partial l= & \left(1-K_{+}\right) g_{2}^{(3)}+\frac{1}{\pi v_{F}}\left(g_{4}^{(1)} g_{1}^{(3)}-2 g_{4}^{(1)} g_{2}^{(3)}+g_{4}^{(3)} g_{1}^{(2)}\right. \\
& \left.-g_{4}^{(3)} g_{2}^{(1)}\right), \\
\partial g_{4}^{(3)} / \partial l= & \left(2-K_{+}-\frac{1}{K_{-}}\right) g_{4}^{(3)}+\frac{1}{\pi v_{F}}\left(-g_{4}^{(3)} g_{4}^{(1)}-2 g_{2}^{(3)} g_{2}^{(1)}\right. \\
& \left.+g_{1}^{(3)} g_{2}^{(1)}+g_{2}^{(3)} g_{1}^{(2)}+g_{1}^{(3)} g_{1}^{(2)}\right)
\end{aligned}
$$

where $l$ stands for (minus) the logarithm of the energy scale measured in units of the high-energy cutoff $E_{c} \sim v_{F} k_{c}$ (of the order of $\sim 0.1 \mathrm{eV}$ ). These equations are similar to those obtained in Ref. 27, except for the nonperturbative improvement of writing the exact dependence of the anomalous dimensions on the forward-scattering couplings (through $K_{+}$ and $K_{-}$). The rest of the equations correspond to the couplings which start having a flow quadratic in backscattering and Umklapp couplings, and they have been already written in Ref. 27.

To illustrate the above ideas, we have solved the full set of scaling equations taking the initial values of the couplings appropriate for individual nanotubes, as listed in Table I. The

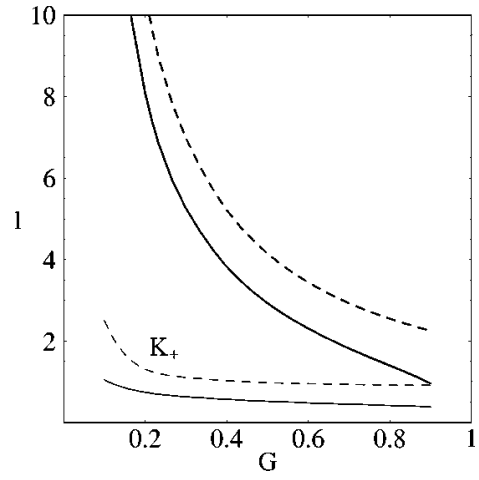

FIG. 4. The thick solid (dashed) line corresponds to the plot of $l=\ln \left(E_{c} / \omega_{0}\right)$, where $\omega_{0}$ is the energy scale at which the parameter $K_{-}$diverges in doped armchair (zigzag) nanotubes, as a function of the effective strength $G=4|g| / \pi v_{F}$ of the phonon-mediated interaction. The lower solid (dashed) curve represents the behavior of the $K_{+}$parameter in doped armchair (zigzag) nanotubes.

strength of the Coulomb potential has been averaged over the length of the nanotube by taking $v=\left(e^{2} / 2 \pi \kappa\right) \log \left|k_{c} / k_{0}\right|$, with $k_{0} \sim 10^{-3} k_{c}$ and a dielectric constant $\kappa$ such that $2 e^{2} / \pi^{2} \kappa v_{F}$ $=1.0$. We have considered in particular the case of doped nanotubes, which is most relevant to establish the comparison with the experimental observations.

The results show that there is always some low-energy scale $\omega_{0}$ at which the charge stiffness $K_{-}$diverges, as represented in Fig. 4. This marks the breakdown of the Luttingerliquid picture, since that effect corresponds to the development of a branch cut in such a physical quantity. For a sensible choice of the effective coupling $4|g| / \pi v_{F} \approx 0.2$, the Luttinger-liquid behavior extends anyhow for about 4 orders of magnitude below the high-energy cutoff $E_{c} \sim 0.1 \mathrm{eV}$, as shown in Fig. 4. The breakdown of the Luttinger-liquid is beyond the range of energies which have been tested experimentally before entering the Coulomb blockade regime of the nanotube. ${ }^{6,7}$ However, the appearance of a new phase characterized by a divergent compressibility should be eventually measured in suitably long samples, or in thinner nanotubes with enhanced electron-phonon interactions. ${ }^{34}$ We have also checked that, in metallic nanotubes with the Fermi level fine tuned to remain at half filling, the low-energy scale $\omega_{0}$ can be significantly enlarged, shrinking the energy range for the existence of Luttinger-liquid behavior. ${ }^{35}$

\section{SCREENING EFFECTS IN CARBON NANOTUBE ROPES}

The above framework has to be conveniently modified in order to account for the electronic properties of the nanotube ropes. These are systems that may consist of a large number of nanotubes, so that the coupling between those with metallic character may have a large effect on the electronic properties. The amplitude for the tunneling of single electrons between neighboring metallic nanotubes is strongly suppressed in ropes made of a random mixture of nanotubes with different helicities. ${ }^{16,17}$ In these conditions, the coupling between metallic nanotubes has essentially electrostatic char- 
acter. Actually, given the long-range nature of the Coulomb interaction, the appropriate description of the rope is made by assuming that the charge in a given nanotube interacts with the charge present in the rest of the metallic nanotubes.

We have to discern between the previously defined couplings $g_{i}^{(j)}$, for the interactions within each metallic nanotube, and new couplings $u_{i}^{(j)}(a, b)$ parametrizing the interactions between different metallic nanotubes labeled by $a$ and $b$ (we will use the same convention for super and subindices in the intratube and the intertube interactions). As pointed out, the main contributions to these couplings come from the repulsive Coulomb interaction in forward-scattering channels. Then, as a first step in the description of the ropes, we can concentrate on the analysis of the model including the $g_{4}^{(4)}$, $g_{2}^{(2)}, g_{2}^{(4)}, g_{4}^{(2)}, u_{4}^{(4)}(a, b), u_{2}^{(2)}(a, b), u_{2}^{(4)}(a, b)$, and $u_{4}^{(2)}(a, b)$ couplings. This already gives rise to relevant effects, as it allows us to capture the different regimes that arise when shifting from small to large number of metallic nanotubes in the rope. We are going to show that, in the latter case, there are remarkable screening effects in the repulsion between two electrons living in the same nanotube, from the interaction with the charge in the other metallic nanotubes.

We can accomplish the solution of the model with forward-scattering interactions in the rope, by an extension of the diagrammatic method used for the solution of the Luttinger model. ${ }^{33}$ By effect of the quantum corrections, the bare couplings $g_{i}^{(j)}$ are dressed to become interaction vertices $D_{i}^{(j)}$, as well as the couplings $u_{i}^{(j)}(a, b)$ are dressed to turn into vertices $V_{i}^{(j)}(a, b)$. The dressed vertices obey the selfconsistent equations represented diagrammatically in Fig. 5. For the intratube vertices $D_{i}^{(j)}$, the equations take the form

$$
\begin{aligned}
D_{4}^{(4)}= & g_{4}^{(4)}+g_{4}^{(4)} \Pi_{+} D_{4}^{(4)}+g_{4}^{(2)} \Pi_{-} D_{4}^{(2)}+g_{2}^{(4)} \Pi_{+} D_{2}^{(4)} \\
& +g_{2}^{(2)} \Pi_{-} D_{2}^{(2)}+\sum_{c \neq a}\left[u_{4}^{(4)}(a, c) \Pi_{+} V_{4}^{(4)}(c, a)\right. \\
& +u_{4}^{(2)}(a, c) \Pi_{-} V_{4}^{(2)}(c, a)+u_{2}^{(4)}(a, c) \Pi_{+} V_{2}^{(4)}(c, a) \\
& \left.+u_{2}^{(2)}(a, c) \Pi_{-} V_{2}^{(2)}(c, a)\right],
\end{aligned}
$$

$$
\begin{aligned}
D_{4}^{(2)}= & g_{4}^{(2)}+g_{4}^{(2)} \Pi_{+} D_{4}^{(4)}+g_{4}^{(4)} \Pi_{-} D_{4}^{(2)}+g_{2}^{(2)} \Pi_{+} D_{2}^{(4)} \\
& +g_{2}^{(4)} \Pi_{-} D_{2}^{(2)}+\sum_{c \neq a}\left[u_{4}^{(2)}(a, c) \Pi_{+} V_{4}^{(4)}(c, a)\right. \\
& +u_{4}^{(4)}(a, c) \Pi_{-} V_{4}^{(2)}(c, a)+u_{2}^{(2)}(a, c) \Pi_{+} V_{2}^{(4)}(c, a) \\
& \left.+u_{2}^{(4)}(a, c) \Pi_{-} V_{2}^{(2)}(c, a)\right],
\end{aligned}
$$

$$
\begin{aligned}
D_{2}^{(4)}= & g_{2}^{(4)}+g_{2}^{(4)} \Pi_{+} D_{4}^{(4)}+g_{2}^{(2)} \Pi_{-} D_{4}^{(2)}+g_{4}^{(4)} \Pi_{+} D_{2}^{(4)} \\
& +g_{4}^{(2)} \Pi_{-} D_{2}^{(2)}+\sum_{c \neq a}\left[u_{2}^{(4)}(a, c) \Pi_{+} V_{4}^{(4)}(c, a)\right. \\
& +u_{2}^{(2)}(a, c) \Pi_{-} V_{4}^{(2)}(c, a)+u_{4}^{(4)}(a, c) \Pi_{+} V_{2}^{(4)}(c, a) \\
& \left.+u_{4}^{(2)}(a, c) \Pi_{-} V_{2}^{(2)}(c, a)\right],
\end{aligned}
$$
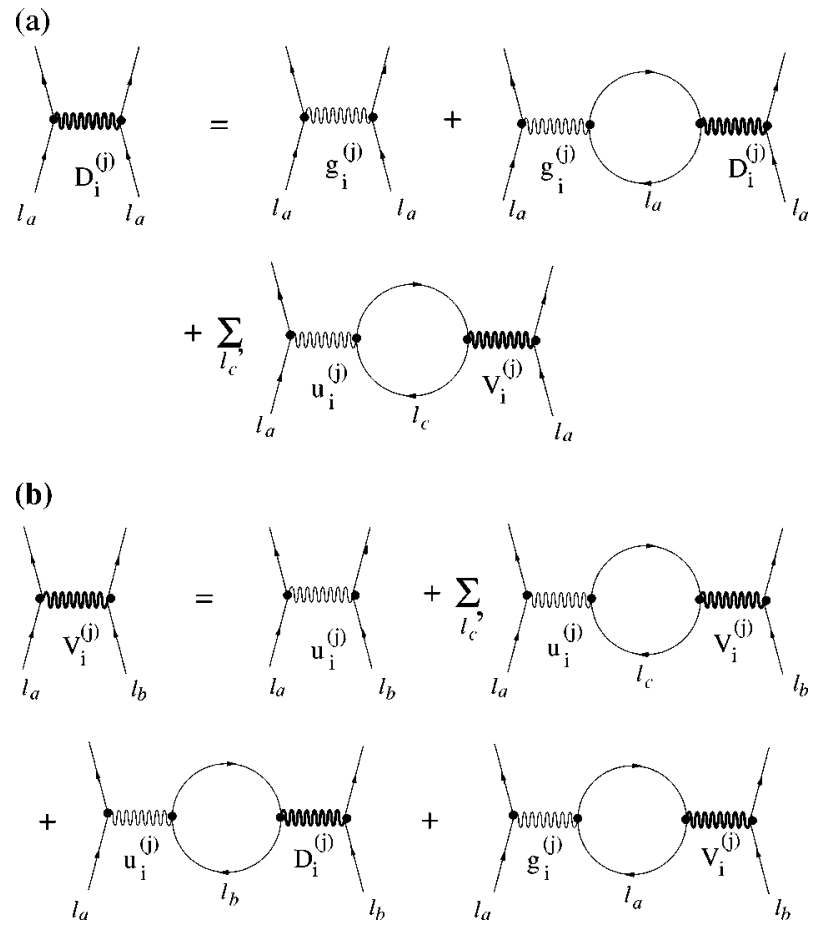

FIG. 5. (a) Diagrams contributing to the screening of Coulomb interactions between currents $l_{a}$ within the same nanotube. The currents have well-defined chirality and spin. The last term takes into account the polarization of the rest of $n-1$ metallic nanotubes in the rope. The prime stands for the exclusion $c \neq a$ in the summation. (b) Diagrams contributing to the screening of Coulomb interactions between currents $l_{a}$ and $l_{b}$ belonging to different metallic nanotubes. The prime stands for the exclusion $c \neq a, b$ in the summation.

$$
\begin{aligned}
D_{2}^{(2)}= & g_{2}^{(2)}+g_{2}^{(2)} \Pi_{+} D_{4}^{(4)}+g_{2}^{(4)} \Pi_{-} D_{4}^{(2)}+g_{4}^{(2)} \Pi_{+} D_{2}^{(4)} \\
& +g_{4}^{(4)} \Pi_{-} D_{2}^{(2)}+\sum_{c \neq a}\left[u_{2}^{(2)}(a, c) \Pi_{+} V_{4}^{(4)}(c, a)\right. \\
& +u_{2}^{(4)}(a, c) \Pi_{-} V_{4}^{(2)}(c, a)+u_{4}^{(2)}(a, c) \Pi_{+} V_{2}^{(4)}(c, a) \\
& \left.+u_{4}^{(4)}(a, c) \Pi_{-} V_{2}^{(2)}(c, a)\right],
\end{aligned}
$$

where $\Pi_{+}$and $\Pi_{-}$are the respective polarizations for right and left branches with linear dispersion, given by

$$
\begin{aligned}
& \Pi_{+}(k, \omega)=\frac{k}{2 \pi\left(\omega-v_{F} k\right)}, \\
& \Pi_{-}(k, \omega)=\frac{-k}{2 \pi\left(\omega+v_{F} k\right)} .
\end{aligned}
$$

The equations giving the intertube vertices $V_{i}^{(j)}(a, b)$ are of the same type as those written above and can be also obtained in a straightforward way.

The resolution of the self-consistent equations produces in general dressed vertices $D_{i}^{(j)}$ and $V_{i}^{(j)}$ that depend on the momentum transfer $k$ and the frequency $\omega$. For the sake of handling a simpler solution allowing to represent the vertices as dressed coupling constants, we will take in what follows the static approximation in the polarizations (39) and (40). The precise form of the solution still depends on the values 
chosen for the bare couplings $g_{i}^{(j)}$ and $u_{i}^{(j)}(a, b)$. In this respect and having in mind the long-range character of the Coulomb interaction, we will assume that the couplings $u_{i}^{(j)}(a, b)$ do not depend on the particular pair of metallic nanotubes, so that we can simply write them as

$$
u_{i}^{(j)}=v
$$

with $v=\left(e^{2} / 2 \pi \kappa\right) \log \left|k_{c} / k_{0}\right|$. For the $g_{i}^{(j)}$ couplings, we will stick to the values summarized in Table I.

In the case of a bundle of $n$ armchair nanotubes, the solution of the self-consistent equations is

$$
\begin{gathered}
D_{4}^{(4)}=D_{2}^{(2)}=v f(n v)+g f(g), \\
D_{2}^{(4)}=D_{4}^{(2)}=v f(n v)-g f(g), \\
V_{i}^{(j)}=v f(n v),
\end{gathered}
$$

where $f(x)=1 /\left(1+4 x / \pi v_{F}\right)$.

In the case of a rope with $n$ metallic zigzag nanotubes, we have

$$
D_{i}^{(j)}=[v f(n v+\delta g)+\delta g] f(\delta g)
$$

for all of the four intratube vertices, and

$$
V_{i}^{(j)}=[v f(n v+\delta g)] f(\delta g)
$$

for the intertube vertices.

There are a number of conclusions from this calculation that have to be emphasized:

(i) As expected, the calculation produces finite renormalizations. The physical process that we are considering at this level is the screening of the Coulomb interaction, described by RPA-like diagrams well-defined in the infrared limit (with no logarithmic divergences).

(ii) The effect of the Coulomb screening is well-captured in the expression of the dressed vertices. The RPA-like diagrams considered screen repulsive interactions and enhance the phonon-mediated interactions, and the latter always prevail for sufficiently large number $n$ of metallic nanotubes since

$$
\lim _{n \rightarrow \infty}\left|D_{i}^{(j)}\right|=\frac{|g|}{1+4 g / \pi v_{F}} .
$$

(iii) For $4|g| / \pi v_{F}$ close to 1 , we find the same kind of divergence already observed in the bosonization picture, ${ }^{14,19}$ which corresponds to the Wentzel-Bardeen singularity. Beyond that limit, to treat the electron-phonon interaction as a perturbation of the purely electronic problem makes no sense and a careful treatment demands to deal with the electronphonon "bound state" (polaron) as the starting point.

(iv) The intertube vertices $V_{i}^{(j)}$ show also the effects of screening of the repulsive interaction. They do not enter explicitly in the expression of any response function, and their influence is not significant anyhow in the massive ropes, as they vanish in the limit or large number $n$ of metallic nanotubes. (v) In the limit $n \rightarrow 0, g \rightarrow 0$, we recover the bare vertices used in precedent studies of superconductivity in isolated nanotubes. ${ }^{28}$

In the ropes, the couplings that give the actual strength of the interaction in the forward-scattering channels correspond to the vertices $D_{4}^{(4)}, D_{2}^{(2)}, D_{2}^{(4)}$, and $D_{4}^{(2)}$. These are therefore the couplings that have to be taken into account when looking for the electronic instabilities in the bundle of nanotubes. The intratube couplings have nontrivial scaling properties that reflect the behavior of the electron system at low energies. Their scaling equations are similar to those derived in Ref. 27 for individual nanotubes, except for the replacement of the couplings $g_{4}^{(4)}, g_{2}^{(2)}, g_{2}^{(4)}$, and $g_{4}^{(2)}$ by the corresponding dressed couplings $D_{4}^{(4)}, D_{2}^{(2)}, D_{2}^{(4)}$, and $D_{4}^{(2)}$, that incorporate the integration of the intertube interactions in the rope. Thus, we have

$$
\partial g_{1}^{(2)} / \partial l=\frac{1}{\pi v_{F}}\left(D_{4}^{(2)}-D_{2}^{(2)}\right) g_{1}^{(2)}+\frac{1}{\pi v_{F}}\left(g_{4}^{(3)} g_{1}^{(3)}-g_{2}^{(1)} g_{1}^{(1)}\right),
$$

$$
\begin{aligned}
\partial g_{1}^{(3)} / \partial l= & \frac{1}{\pi v_{F}}\left(D_{4}^{(2)}+D_{2}^{(2)}\right) g_{1}^{(3)}+\frac{1}{\pi v_{F}}\left(-2 g_{1}^{(3)} g_{1}^{(1)}+g_{2}^{(3)} g_{1}^{(1)}\right. \\
& \left.+g_{4}^{(3)} g_{1}^{(2)}\right),
\end{aligned}
$$

$$
\begin{aligned}
\partial g_{2}^{(1)} / \partial l= & \frac{1}{\pi v_{F}}\left(D_{4}^{(2)}-D_{2}^{(2)}\right) g_{2}^{(1)}+\frac{1}{\pi v_{F}}\left(g_{4}^{(1)} g_{1}^{(2)}-2 g_{4}^{(1)} g_{2}^{(1)}\right. \\
& \left.+g_{4}^{(3)} g_{1}^{(3)}-g_{4}^{(3)} g_{2}^{(3)}-g_{1}^{(2)} g_{1}^{(1)}\right)
\end{aligned}
$$

$$
\begin{aligned}
\partial g_{2}^{(3)} / \partial l= & \frac{1}{\pi v_{F}}\left(D_{4}^{(2)}+D_{2}^{(2)}\right) g_{2}^{(3)}+\frac{1}{\pi v_{F}}\left(g_{4}^{(1)} g_{1}^{(3)}-2 g_{4}^{(1)} g_{2}^{(3)}\right. \\
& \left.+g_{4}^{(3)} g_{1}^{(2)}-g_{4}^{(3)} g_{2}^{(1)}\right)
\end{aligned}
$$

$$
\begin{aligned}
\partial g_{4}^{(3)} / \partial l= & \frac{1}{\pi v_{F}} 2 D_{4}^{(2)} g_{4}^{(3)}+\frac{1}{\pi v_{F}}\left(-g_{4}^{(3)} g_{4}^{(1)}-2 g_{2}^{(3)} g_{2}^{(1)}+g_{1}^{(3)} g_{2}^{(1)}\right. \\
& \left.+g_{2}^{(3)} g_{1}^{(2)}+g_{1}^{(3)} g_{1}^{(2)}\right)
\end{aligned}
$$

where $l$ stands again for (minus) the logarithm of the energy scale in units of the high-energy cutoff $E_{c}$. The rest of the equations are completely similar to those written in Ref. 27, again with the replacement of the forward-scattering couplings by the corresponding dressed couplings.

The discussion of the low-energy properties that derive from the scaling equations leads to a very rich phase diagram, since the initial values of the couplings $D_{4}^{(4)}, D_{2}^{(2)}, D_{2}^{(4)}$, and $D_{4}^{(2)}$ depend on the number of metallic nanotubes in the rope through Eqs. (42), (43), and (45). In order to unveil the different phases, we have also to pay attention to the scaling of the amplitudes for intertube hopping, which will give a measure of the development of 3D coherence in the rope.

\section{INTERTUBE PAIR HOPPING AND COHERENCE}

Maarouf, Kane, and Mele ${ }^{16}$ have elaborated a microscopic tight-binding theory of single-electron tunneling between 
two carbon nanotubes with arbitrary helicities. According to them, the tunneling amplitude $t_{T}$ between two nanotubes with the same helicity and radius $R$ is

$$
t_{T}=t_{G} \sqrt{\frac{a_{0}}{4 \pi R}},
$$

where $t_{G}$ is the amplitude for transverse hopping between two graphite sheets and $a_{0}$ is the length scale associated with the hopping between carbon atoms. The mismatch of the lattices of parallel nanotubes with different helicity makes difficult the conservation of the longitudinal momentum in the tunneling process, producing an exponential suppression of the tunneling amplitude ${ }^{16}$

$$
t_{\perp}=t_{T} e^{-(1 / 4) R a_{0} \delta k^{2}},
$$

where $\delta k$ is the momentum mismatch of the Fermi points in the two nanotubes, arising from the different orientation of the respective carbon lattices. This introduces a factor of suppression that sets the single-particle intertube hopping about three orders of magnitude below the estimate for $t_{T}{ }^{17}$ The authors of Ref. 16 applied these results to a compositionally disordered rope (a mixture of nanotubes with random helicities). In this kind of system, even if all the nanotubes are metallic, it has been shown that the localization length in the transverse direction is $\xi_{\perp} \sim 1 \mathrm{~nm}$. One may conclude that the single-electron states are not extended throughout the compositionally disordered rope, but localized in individual nanotubes. ${ }^{16}$

Such a small value of $\xi_{\perp}$ acts as a cutoff in the scaling equation for the single-electron tunneling amplitude $t_{\perp}$, given in terms of the charge stiffness $K$ of the $1 \mathrm{D}$ electron liquids by ${ }^{36}$

$$
\partial t_{\perp} / \partial l=\left(2-\frac{K+K^{-1}}{2}\right) t_{\perp} .
$$

Then, $t_{\perp}$ can be considered as a constant in our low-energy analysis, at scales beyond the localization length. On the other hand, compositional disorder does not interfere with the tunneling of Cooper pairs in as much they are preformed with zero total momentum. We still can have quantum coherent behavior in the transverse direction beyond $\xi_{\perp}$, if the amplitude for pair hopping grows at low energies.

The fact that a rope is made of nanotubes with different helicities and diameters has another consequence regarding its electronic properties. It prevents the formation of any phase with spin-density-wave or charge-density-wave order at the rope level. On the other hand, the nanotubes may have strong SC correlations. The transport of the Cooper pairs along each nanotube is governed by the anomalous dimension of the 1D two-particle propagator

$$
\Delta=-\frac{1}{\pi v_{F}}\left(D_{2}^{(2)} \pm g_{1}^{(1)}+g_{2}^{(1)} \pm g_{1}^{(2)}\right),
$$

where the upper sign holds for singlet pairing and the lower sign for triplet pairing. The SC response function for a single nanotube $\mathcal{R}^{(\|)}(\omega)$ does not scale itself homogeneously at low energies, but its derivative

$$
\overline{\mathcal{R}}^{(\|)}(\omega)=\frac{\partial \mathcal{R}^{(\|)}(\omega)}{\partial \ln \omega}
$$

satisfies the scaling equation ${ }^{33}$

$$
\partial \overline{\mathcal{R}}^{(\|)} / \partial l=\Delta \overline{\mathcal{R}}^{(\|)} .
$$

The interactions corresponding to $g_{1}^{(1)}$ and $g_{1}^{(2)}$ are always attractive, pointing at the enhancement of the SC correlations with $s$-wave symmetry at low energies.

In the compositionally disordered ropes, the coherence is established by the tunneling of Cooper pairs between neighboring metallic nanotubes. The amplitude $J$ for this process has a dependence on the energy scale characterized again by the anomalous dimension $\Delta$. The scaling equation for $J$ is

$$
\partial J / \partial l=\Delta J+c t_{\perp}^{2},
$$

where $c$ is a parameter depending on the charge stiffness in the metallic nanotubes. ${ }^{36}$

We have characterized the SC transition by looking for a pole $\omega_{c}$ in the 3D two-particle propagator $\mathcal{R}(\omega)$ of the Cooper pairs along the rope. This is related to the SC response function $\mathcal{R}^{(\|)}(\omega)$ in the individual nanotubes through the Schwinger-Dyson equation

$$
\mathcal{R}(\omega)=\frac{\mathcal{R}^{(\|)}(\omega)}{1-\mathcal{R}^{(\|)}(\omega) \tilde{J}},
$$

where $\widetilde{J}$ is the (dimensionless) pair hopping measured in units of the high-energy cutoff. The critical frequency can be estimated in the SC phase by solving the equation for the pole in the $3 \mathrm{D}$ Cooper pair propagator

$$
\mathcal{R}^{(\|)}\left(\omega_{c}\right)=1 / \tilde{J}
$$

Moreover, we have studied the competition between superconductivity and other charge instabilities in the rope by rebosonizing the system at each step in the integration of the scaling equations for the couplings, considering backscattering and Umklapp scattering as small perturbations. The individual nanotubes are characterized now by the independent velocities

$$
\begin{aligned}
& v_{N \pm}=v_{F}+\frac{D_{4}^{(4)} \pm D_{2}^{(4)}}{\pi}+\frac{D_{2}^{(2)} \pm D_{4}^{(2)}}{\pi}, \\
& v_{J \pm}=v_{F}+\frac{D_{4}^{(4)} \pm D_{2}^{(4)}}{\pi}-\frac{D_{2}^{(2)} \pm D_{4}^{(2)}}{\pi}
\end{aligned}
$$

The corresponding Luttinger liquid parameters are

$$
K_{ \pm}=\sqrt{\frac{\pi v_{F}+\left(D_{4}^{(4)} \pm D_{2}^{(4)}\right)-\left(D_{2}^{(2)} \pm D_{4}^{(2)}\right)}{\pi v_{F}+\left(D_{4}^{(4)} \pm D_{2}^{(4)}\right)+\left(D_{2}^{(2)} \pm D_{4}^{(2)}\right)}} .
$$

We have obtained complementary information about the physical properties of the system by studying the regions where the $K_{ \pm}$parameters either vanish or diverge. This happens when one of the functions given by Eq. (64) develops a branch cut at some energy scale, what has to be understood as the onset of a phase transition in the system. 


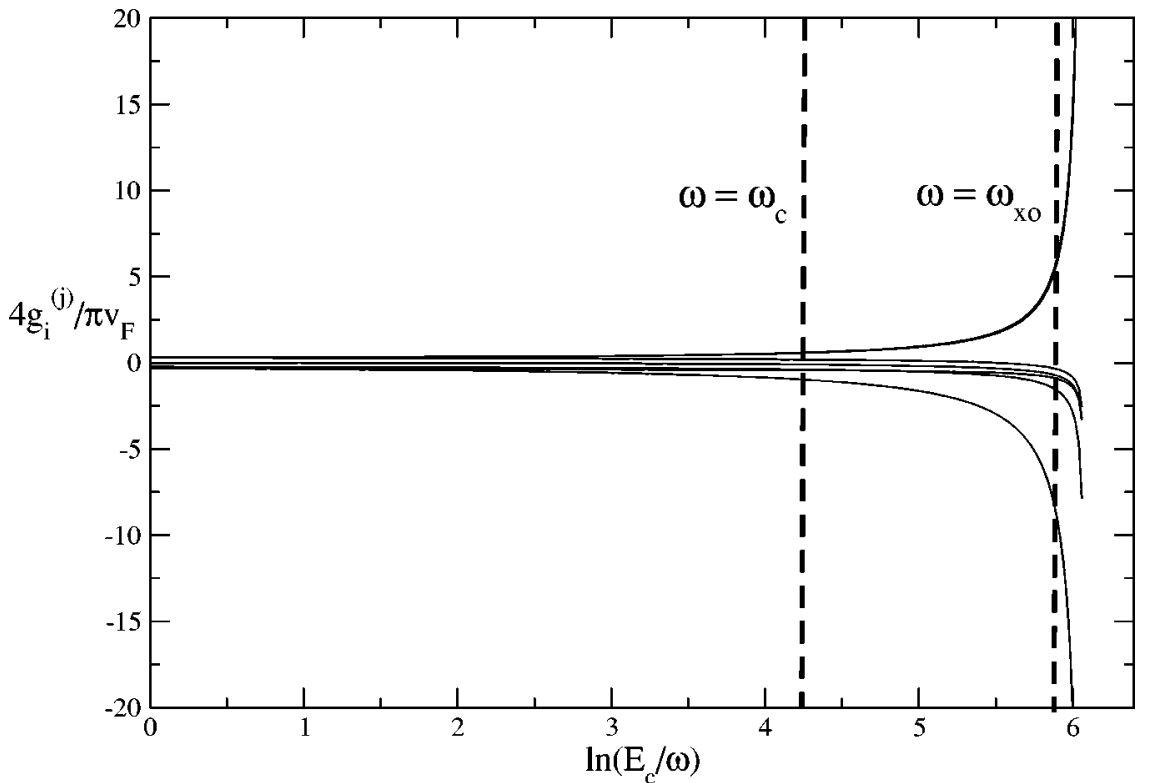

FIG. 6. Scale dependence of the coupling constants for $4|g| / \pi v_{F}=0.3$ in a bundle with $n$ $=200$ undoped metallic zigzag nanotubes. For these parameters the system is in the SC state with a critical frequency $\omega_{c}$ shown in the figure. At that point, the scaling stops due to the opening of the SC single-particle gap. Here we have continued the flow to show that the system at $\omega_{c}$ is well inside the weak-coupling regime. The crossover to the strong-coupling regime would take place at a lower frequency $\omega_{x o}$.

\section{RESULTS}

Before going to a detailed description of the phase diagrams of armchair and zigzag nanotubes, we would like to discuss first the nature of the two fixed points that we find in this problem. Within our computational approach we can formally set to zero all the backscattering and Umklapp couplings, keeping the system at a nontrivial fixed point described in the bosonization picture. ${ }^{14}$ In that case, we can say that the system is in the weak-coupling limit because none of the couplings is large. However, that fixed point is in general unstable against chirality-breaking perturbations such as backscattering and Umklapp processes, that we know are important in this problem because there is significant electron-phonon coupling in the individual nanotubes. Under these perturbations, the system moves away from the original bosonization fixed point and approaches a strong-coupling fixed point. However, the crossover behavior is very slow, being characterized by a relatively small crossover frequency $\omega_{\mathrm{xo}}$.

Moreover, in a large region of the phase diagram the scaling as $\ln \left(E_{c} / \omega\right) \rightarrow \infty$ is cutoff by the onset of the 3D coherence in the transport of the Cooper pairs. This opens a gap in the single-particle spectrum before $\omega_{\text {xo }}$ has been reached (see Fig. 6). In these conditions, the SC phase remains in the region of influence of the weak-coupling fixed point, which governs the main physical properties. If $\omega_{c} \gg \omega_{\text {xо }}$, the description of a SC transition (two-particle propagators, gaps, critical temperatures) obtained using the exact solution at the bosonization fixed point is essentially correct.

A different way to study the development of SC correlations in individual nanotubes is to look for the divergence in the SC response function $\mathcal{R}^{(\|)}(\omega){ }^{27,28}$ That effect is attached to the strong-coupling regime and has completely different physical origin. This manifest itself in several features as, for instance, that the critical frequency increases with the number $n$ of metallic nanotubes in a rope in the weak-coupling SC phase (see Fig. 7) while stays almost constant or decreases in the strong-coupling one. Another important differ- ence that we find between the weak and strong-coupling regimes is the scale dependence of the Luttinger-liquid parameters. While in the weak-coupling regime $K_{+}$and $K_{-}$ hardly renormalize, in the strong-coupling regime they can vanish or diverge as we integrate out the high-energy degrees of freedom. This behavior is very much like that of a 1D interacting electron liquid, with the Luttinger-liquid behavior and its instabilities.

The different phases which arise in this approach have been represented in Figs. 8 and 9, where contour lines of constant $l_{c} \equiv \ln \left(E_{c} / \omega_{c}\right)$ have been also plotted. The critical frequency $\omega_{c}$ represents in either case the value at which the $\mathrm{SC}$ transition takes place or the point where any of the Luttinger-liquid parameters develops a branch cut, as the scaling equations [including Eqs. (58) and (59)] are solved at progressively low energies. We have discerned between the different cases of zigzag and armchair nanotubes and whether they are doped or not, as the initial conditions for the scaling equations of the couplings are different in each case.

The common trend observed in the four phase diagrams is given by the boundary that separates the SC phase from the region with singular behavior of any of the Luttinger-liquid parameters at small and moderate values of $|g|$. This line is actually very close to the boundary of the region in which the pair-hopping amplitude does not grow large at low energies. When this happens, the nanotubes in the rope start to behave as uncoupled 1D systems, what explains the disappearance of the SC transition. This provides a graphic representation of the distinction we have made between the regime of weakcoupling superconductivity and the strong-coupling regime given by the instabilities of the individual nanotubes.

In the undoped ropes, we observe that the latter correspond to the vanishing of $K_{+}$at small values of $|g|$. A closer look at the solution of the equations reveals that the velocity $v_{J+}$ given by Eq. (63) is the parameter being driven to zero. The physical effect corresponds then to the vanishing of the Drude peak $D_{+}$, according to Eq. (26). This new phase has to be interpreted as the extension of the Mott insulating phase 


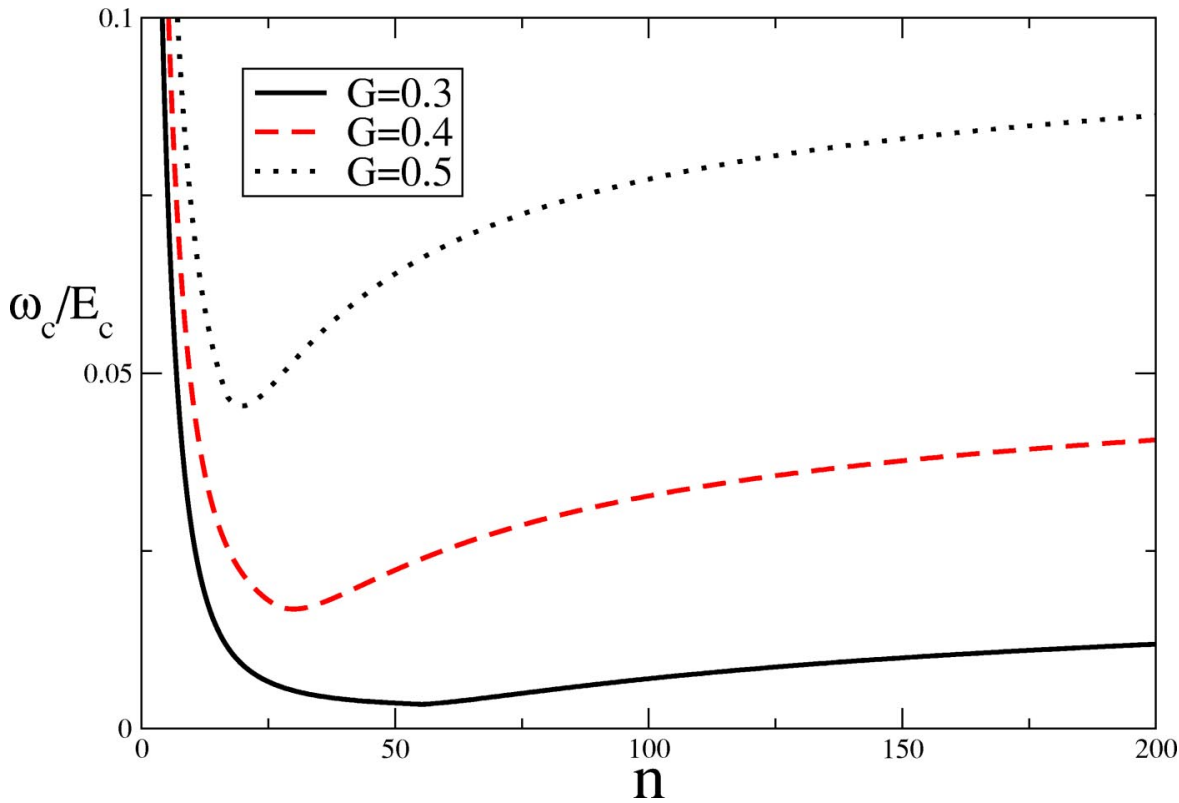

FIG. 7. Dependence of the critical frequency in a bundle with $n$-undoped metallic zigzag nanotubes for $4|g| / \pi v_{F}=0.3$ (solid), 0.4 (dashed), and 0.5 (dotted). The increase of $\omega_{c}$ with $n$ marks the setting of the SC phase. That kind of behavior, also observed in experimental samples, is characteristic of weak-coupling superconductivity (Ref. 14). found in the studies of carbon nanotubes with repulsive interactions. $^{4,5}$ In our case, it is favored in the undoped systems, as the effective interaction from phonon exchange shows a repulsive component in some of the interaction channels of the carbon nanotubes at half filling.

In doped nanotube ropes, the phase that prevails in the absence of coherent pair hopping corresponds to the divergence of $K_{+}$at moderate and large values of $n$, as observed in Figs. 8 and 9. This singular behavior stems now from the vanishing of the renormalized $v_{N+}$ velocity in Eq. (62). Therefore, the onset of the new phase is characterized on physical grounds by the divergence of the compressibility $\kappa_{+}$ in the channel of the total charge. Again, these effects have to be understood in terms of the transition to a regime marked by the decoupling of the different metallic nanotubes in the rope.

For low content of metallic nanotubes or values of the effective coupling $G=4|g| / \pi v_{F}$ close to 1 , we see that the natural tendency of the doped system is to fall into a phase given by the divergence of the $K_{-}$parameter. The onset of this phase is characterized then by the divergence of the compressibility in the channel of the mismatch of charge in the two gapless subbands. For values of $G$ close to 1 , it becomes clear that the divergence has the same character of the Wentzel-Bardeen singularity, which corresponds in our description to the pole found in the $f(g)$ component of the dressed couplings (42), (43), and (45). That kind of phase is the natural counterpart of the SC instability since, for sufficiently strong attraction, the system finds more favorable its macroscopic segregation than the formation of Cooper pairs. ${ }^{34}$ From a 1D point of view, the parameters $K_{+}$and $K_{-}$ give the strength of the uniform density-density correlations in their respective channels. ${ }^{32,33}$ The divergence of $K_{-}$marks then the approach of a regime with increasingly large density fluctuations. The singularity reflects the fact that the theoretical framework applied breaks down at certain point, beyond which the variables chosen do not allow the description of the rope as a homogeneous system.

\section{CONCLUSIONS}

We have shown that a variety of zero-temperature phases are possible in the carbon nanotube ropes, depending mainly on the doping level, the number of metallic nanotubes, and the strength of the electron-phonon interaction (which is specially sensitive to the diameter of the individual nanotubes). Our results point at the existence of a SC phase extended over a large region of the phase diagram, given in terms of the effective coupling for the phonon-mediated interaction and the number of metallic nanotubes in the rope. There are two effects that cooperate to make possible the superconductivity of the nanotube ropes. One of them is the reduction in the strength of the long-range Coulomb repulsion, which becomes more suppressed for larger contents of metallic nanotubes in a rope. This allows us to explain the appearance of large SC correlations in the thick ropes at low temperatures. The other factor has to do with the fact that once the SC correlations develop, the transport of the Cooper pairs is also enhanced along the transverse directions of the rope. The intertube hopping of Cooper pairs becomes actually the dominant tunneling amplitude at low energies, in typical ropes made of a random mixture of nanotubes with different helicities. We have made clear with our analysis that the superconductivity is a phenomenon that belongs to the weakcoupling regime in sufficiently thick ropes, as it springs from a suitable reduction of the Coulomb repulsion that makes the phonon-mediated effective interaction to prevail in the individual nanotubes.

The plausibility of the SC phase in the nanotube ropes is the most significant result of our study, given the implications that it has from the phenomenological point of view. The existence of SC transitions has been reported in Refs. 11 and 12 , in experiments carried out in samples with an approximate content of about 100 metallic nanotubes. The transition temperatures that have been measured are slightly below $0.5 \mathrm{~K}$. When converted to energy units, that scale turns out to be of the order of $\sim 10^{-3}$ times our high-energy cutoff 

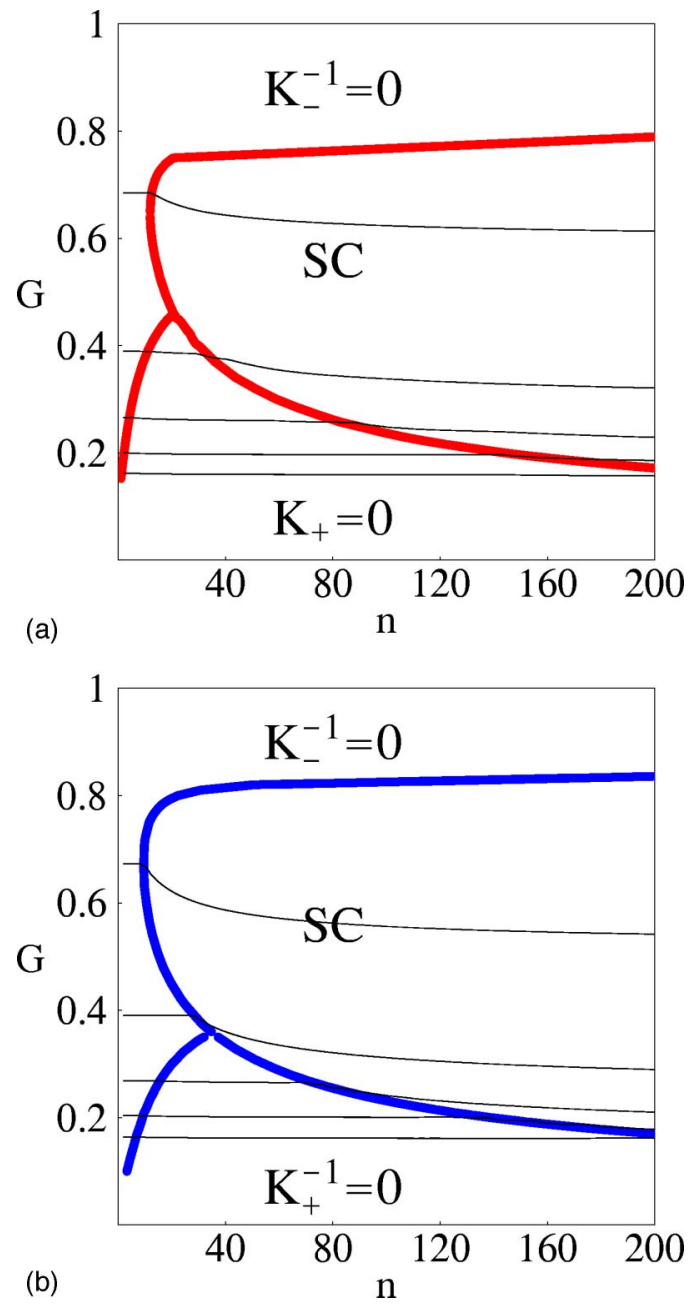

FIG. 8. Phase diagram of undoped (a) and doped (b) armchair nanotubes as a function of the number of metallic nanotubes $n$ in the rope and the strength of the attractive interaction $G$ $=4|g| / \pi v_{F}$. Thick lines represent the phase boundaries between a $3 \mathrm{D}$ phase-coherent SC phase and the $1 \mathrm{D}$ phases characterized by the breaking of the parameters $K_{+}$and $K_{-}$. Thin lines are contours of constant critical frequency $\omega_{c}$, starting from above with $\ln \left(E_{c} / \omega_{c}\right)$ $=2,4,6, \ldots$.

$E_{c}$. Therefore, we can infer that the samples where the SC transitions have been observed should correspond to points in the phase diagram with $\ln \left(E_{c} / \omega_{c}\right) \approx 7$. Taking the phase diagram of the doped zigzag nanotubes as a most appropriate guide, we see that the points for the mentioned samples seem to be sitting near the boundary between the SC phase and the phase with the $1 \mathrm{D}$ charge instability.

The temperature of the transition to the SC phase may be also affected by factors that have to do with the internal structure of the rope, in particular with the way the percolation of the Cooper pairs takes place in the transverse section of the rope. In any event, a distinctive prediction of our theoretical construction is that the SC transition has to disappear below a certain number of metallic nanotubes in the ropes. This is consistent with the experimental observations reported in Ref. 12, where it has been shown how the transition is not completed in a sample with relatively low resistance
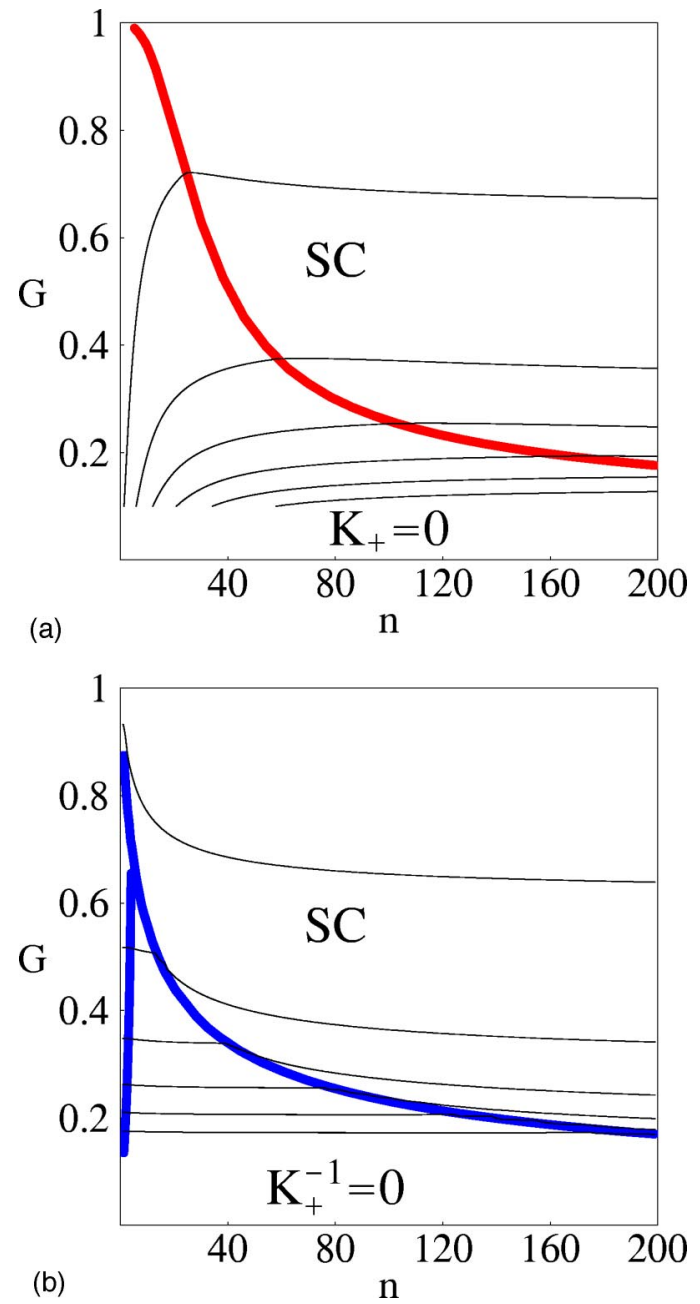

FIG. 9. Phase diagram of undoped (a) and doped (b) zigzag nanotubes as a function of the number of metallic nanotubes $n$ in the rope and the strength of the attractive interaction $G$ $=4|g| / \pi v_{F}$. Thick and thin lines have the same meaning as in Fig. 8 . In the case of doped nanotubes, the factor $\delta$ of reduction of the effective interactions in Table I has been set to 0.1 . The narrow phase to the left of diagram (b) corresponds to the divergence of the $K_{\text {- parameter. }}$

and with a total of about 45 nanotubes. Looking for phenomenological signatures in the opposite case of very thick ropes, the phase diagrams in Figs. 8 and 9 suggest that higher transition temperatures could be obtained by dealing with samples with larger number of metallic nanotubes. The newly devised techniques to separate metallic and semiconducting nanotubes could be used eventually to produce ropes with higher content of metallic nanotubes. The phase diagrams we have obtained imply that, in a rope assembled with a total of 300 metallic nanotubes, the transition temperature could be increased by a factor of 10 with respect to those observed in Ref. 12. Higher values can be also expected from the enhancement of the Cooper pair tunneling amplitude between the different nanotubes.

To summarize, the nanotube ropes may show quite different transport properties depending on their content of metallic nanotubes. Only above a certain number of them it is 
likely the observation of SC properties. For lower content of metallic nanotubes, there may exist exotic phases with large compressibilities, which belong to the strong-coupling regime characterized by the decoupling of the nanotubes. These phases may appear at temperatures that are below $1 \mathrm{~K}$, so they have the chance to manifest only in samples where their effects are not overshadowed by those of Coulomb blockade. We have seen that before that low-temperature strong-coupling regime is reached, the transport properties have to be given by the Luttinger-liquid behavior, which is the counterpart to the weak-coupling SC phase, in the case of thin ropes dominated by the Coulomb interaction.

\section{ACKNOWLEDGMENTS}

J. V. A. acknowledges support from S. Moukouri and stimulating discussions with J. Allen. J. G. acknowledges financial support from the Ministerio de Ciencia y Tecnología Grant No. BFM2003-05317.
${ }^{1}$ R. Saito, M. Fujita, G. Dresselhaus, and M. S. Dresselhaus, Appl. Phys. Lett. 60, 2204 (1992); J. W. Mintmire, B. I. Dunlap, and C. T. White, Phys. Rev. Lett. 68, 631 (1992); N. Hamada, S. Sawada, and A. Oshiyama, ibid. 68, 1579 (1992).

${ }^{2}$ L. Balents and M. P. A. Fisher, Phys. Rev. B 55, R11 973 (1997).

${ }^{3}$ R. Egger and A. O. Gogolin, Phys. Rev. Lett. 79, 5082 (1997); Eur. Phys. J. B 3, 281 (1998).

${ }^{4}$ C. Kane, L. Balents, and M. P. A. Fisher, Phys. Rev. Lett. 79, 5086 (1997).

${ }^{5}$ H. Yoshioka and A. A. Odintsov, Phys. Rev. Lett. 82, 374 (1999); Phys. Rev. B 59, R10 457 (1999).

${ }^{6}$ M. Bockrath, D. H. Cobden, J. Lu, A. G. Rinzler, R. E. Smalley, L. Balents, and P. L. McEuen, Nature (London) 397, 598 (1999).

${ }^{7}$ Z. Yao, H. W. Ch. Postma, L. Balents, and C. Dekker, Nature (London) 402, 273 (1999).

${ }^{8}$ A. Yu. Kasumov, R. Deblock, M. Kociak, B. Reulet, H. Bouchiat, I. I. Khodos, Yu. B. Gorbatov, V. T. Volkov, C. Journet, and M. Burghard, Science 284, 1508 (1999).

${ }^{9}$ A. F. Morpurgo, J. Kong, C. M. Marcus, and H. Dai, Science 286, 263 (1999).

${ }^{10}$ J. González, Phys. Rev. Lett. 87, 136401 (2001).

${ }^{11}$ M. Kociak, A. Yu. Kasumov, S. Guéron, B. Reulet, I. I. Khodos, Yu. B. Gorbatov, V. T. Volkov, L. Vaccarini, and H. Bouchiat, Phys. Rev. Lett. 86, 2416 (2001).

${ }^{12}$ A. Kasumov, M. Kociak, M. Ferrier, R. Deblock, S. Guéron, B. Reulet, I. Khodos, O. Stéphan, and H. Bouchiat, Phys. Rev. B 68, 214521 (2003).

${ }^{13}$ Z. K. Tang, L. Zhang, N. Wang, X. X. Zhang, G. H. Wen, G. D. Li, J. N. Wang, C. T. Chan, and P. Sheng, Science 292, 2462 (2001).

${ }^{14}$ J. González, Phys. Rev. Lett. 88, 076403 (2002); Phys. Rev. B 67, 014528 (2003).

${ }^{15}$ J. V. Alvarez and J. González, Phys. Rev. Lett. 91, 076401 (2003).

${ }^{16}$ A. A. Maarouf, C. L. Kane, and E. J. Mele, Phys. Rev. B 61, 11156 (2000).
${ }^{17}$ H. Stahl, J. Appenzeller, R. Martel, Ph. Avouris, and B. Lengeler, Phys. Rev. Lett. 85, 5186 (2000).

${ }^{18}$ A. A. Abrikosov, L. P. Gorkov, and I. E. Dzyaloshinski, Methods of Quantum Field Theory in Statistical Physics (Dover, New York, 1975), Chap. 7.

${ }^{19}$ D. Loss and T. Martin, Phys. Rev. B 50, 12160 (1994).

${ }^{20}$ D. Sánchez-Portal, E. Artacho, J. M. Soler, A. Rubio, and P. Ordejón, Phys. Rev. B 59, 12678 (1999).

${ }^{21}$ L. M. Woods and G. D. Mahan, Phys. Rev. B 61, 10651 (2000).

${ }^{22}$ R. Saito, A. Jorio, A. G. Souza Filho, G. Dresselhaus, M. S. Dresselhaus, and M. A. Pimenta, Phys. Rev. Lett. 88, 027401 (2002).

${ }^{23}$ R. A. Jishi, M. S. Dresselhaus, and G. Dresselhaus, Phys. Rev. B 48, 11385 (1993).

${ }^{24}$ L. Pietronero, S. Strässler, H. R. Zeller, and M. J. Rice, Phys. Rev. B 22, 904 (1980).

${ }^{25}$ J. González, F. Guinea, and M. A. H. Vozmediano, Nucl. Phys. B 406, 771 (1993).

${ }^{26}$ J. González, Eur. Phys. J. B 36, 317 (2003).

${ }^{27}$ Yu. A. Krotov, D.-H. Lee, and S. G. Louie, Phys. Rev. Lett. 78, 4245 (1997).

${ }^{28}$ A. Sédéki, L. G. Caron, and C. Bourbonnais, Phys. Rev. B 65, 140515 (2002).

${ }^{29}$ R. Egger and H. Grabert, Phys. Rev. Lett. 79, 3463 (1997).

${ }^{30}$ S. Bellucci and J. Gonzalez, Eur. Phys. J. B 18, 3 (2000).

${ }^{31}$ D. W. Wang, A. J. Millis, and S. Das Sarma, Phys. Rev. B 64, 193307 (2001).

${ }^{32}$ V. J. Emery, in Highly Conducting One-Dimensional Solids, edited by J. T. Devreese, R. P. Evrard, and V. E. Van Doren (Plenum, New York, 1979).

${ }^{33}$ J. Sólyom, Adv. Phys. 28, 201 (1979).

${ }^{34}$ A. De Martino and R. Egger, Phys. Rev. B 67, 235418 (2003).

${ }^{35}$ A. A. Nersesyan and A. M. Tsvelik, Phys. Rev. B 68, 235419 (2003).

${ }^{36}$ V. M. Yakovenko, Pis'ma Zh. Eksp. Teor. Fiz. 56, 523 (1992) [JETP Lett. 56, 510 (1992)]. 